\title{
Prostatectomía radical laparoscópica extraperitoneal: Resultados de una curva de aprendizaje después de 310 casos*
}

\author{
Drs. OCTAVIO CASTILLO C. ${ }^{1,2}$, MANUEL DÍAZ C. ${ }^{1}$, IVAR VIDAL M. ${ }^{1}$, \\ ALEJANDRO FONERÓN V. ${ }^{1}$, RAFAEL SÁNCHEZ-SALAS ${ }^{1}$, GONZALO VITAGLIANO ${ }^{1}$
}

Unidad de Endourología y Laparoscopía Urológica, Clínica Indisa.

2 Departamento de Urología, Facultad de Medicina Oriente, Universidad de Chile. Santiago, Chile.

\begin{abstract}
Extraperitoneal laparoscopic radical prostatectomy experience in 310 patients

Background: Laparoscopic prostatectomy is one of the standard treatments for localized prostate cancer. Aim: To report our experience with extraperitoneal laparoscopic radical prostatectomy (ELRP). Material and Methods: Prospective recording of 310 ELRP performed between 2001 and 2005. The series was divided chronologically in three groups. Group 1 was formed by the first 100 operated patients and groups 2 and 3 by subsequent series of 100 subjects, to show the learning curve. ELRP was performed using an anterograde technique, placing five trocars. Results: Median age of patients was 62 years. No differences in the clinical data of patients in the different groups were observed. Median prostate specific antigen was $13.3 \mathrm{ng} / \mathrm{ml}$. One hundred eighty patients $(60 \%)$ had a Gleason score of 2 to $6: 80$ patients (25\%) had a score of 7 and 50 patients $(15 \%)$ had a score of 8 to 10 . Two hundred fourteen patients $(69 \%)$ were in clinical stage T1c, 62 patients (20\%) were in stage T2a and 34 patients (11\%) were in stage T2b. Surgical times were 185, 139 and $134 \mathrm{~min}$ in groups 1,2 and 3 respectively $(\mathrm{p}<0.05)$. Blood transfusions were required in 20,22 and 3\% of patients in groups 1, 2 and 3 respectively. The figures for blood loss were 624, 451 and $268 \mathrm{cc}$, respectively. Positive surgical margins were observed in 24, 9 and $12 \%$ of patients in groups 1, 2 and 3 respectively. Rectal injuries occurred in 9,2 and none patient of groups 1, 2 and 3 respectively. Urinary fistula occurred in 4, 1 and no patient of groups 1, 2 and 3 respectively. Late complications such as bladder neck stricture and incontinence decreased in consecutive groups. Conclusions: ELRP is a safe and effective technique for the treatment of localized prostate cancer, but it has a learning curve to reduce the incidence of complications.
\end{abstract}

Key words: Extraperitoneal laparoscopic prostatectomy, prostate cancer, learning curve.

*Recibido el 27 de Febrero de 2008 y aceptado para publicación el 23 de Junio de 2008.

No existe ningún interés financiero o comercial en este manuscrito.

Correspondencia: Dr. Octavio Castillo C.

Av. Apoquindo 3990, Of. 809, Las Condes.

Santiago de Chile.

Fax: (56-2) 2282524

E-mail: octaviocastillo@vtr.net 


\section{Resumen}

Objetivo: La Prostatectomía Radical Laparoscópica Extraperitoneal (PRLE) es un procedimiento difícil con una curva de aprendizaje estable comunicada entre 50-100 casos. Intentamos determinar la curva de aprendizaje con un cirujano experimentado en cirugía laparoscópica previamente. Material y Método: Comparamos los resultados operatorios tras 310 casos de PRLE realizada por un único cirujano en cinco años. Las serie se divide en tres grupos: Grupo 1 los 100 primeros pacientes, grupo 2 los siguientes 100 pacientes y el grupo 3 los últimos 110 pacientes. Análisis Estadístico: Se realiza comparación de pares utilizando el test de $\chi^{2}$, considerando $\mathrm{p}<0,05$ estadísticamente significativa. Resultados: Edad media de la serie es de 62,4 años. Mediana de PSA $=13,3 \mathrm{ng} / \mathrm{ml}$, distribución del Gleason de la biopsia: Gleason 2-6 $=180$ pacientes $(60 \%) ; 7$ en 80 pacientes (25\%) y 8-10 en 50 pacientes (15\%). Estadio clínico T1c: 214 pacientes (69\%); T2a en 62 pacientes $(20 \%)$ y T2b in 34 (11\%). No existen diferencias en cuanto a la distribución entre los grupos. Estadio Patológico: T2 en 41\% de los casos. No hubo diferencias entre los 3 grupos en el Gleason quirúrgico, estadio patológico o peso de la glándula. Hubo diferencias significativas en el tiempo operatorio $(\mathrm{p}<0,05)$ entre el grupo 1 y 2-3 pero no entre los grupos 2 y 3, ( Grupo 1: 184,7 min; Grupo 2: 138,9 min; Grupo 3: 134,3 min) y en los márgenes quirúrgicos en pT2, que disminuyeron (Grupo 1: 24\%; Grupo 2: 9\%; Grupo 3: 11,8\%) y en el porcentaje de transfusión (Grupo 1: 20\%; Grupo 2: 22\%; Grupo 3: 2,7\%) $(\mathrm{p}<0,05$ ), así como en la pérdida sanguínea entre los grupos 1 y 2 con respecto al tres: Grupo 1: $624 \mathrm{cc}$; Grupo 2: 451 cc; Grupo 3: $268 \mathrm{cc}$. Las complicaciones quirúrgicas, como lesión del recto o fístula recto-urinaria disminuyeron entre los grupos (lesión rectal Grupo 1: 9 pacientes; Grupo 2: 2 pacientes; Grupo 3: 0 pacientes). Las complicaciones tardías como la esclerosis del cuello vesical también mejoraron con el tiempo. Esclerosis del cuello vesical: Grupo 1: 8 pacientes; Grupo 2: 5 pacientes. Así como mejoraron los resultados de la continencia con el tiempo. Continencia inmediata (tras retirada de catéter) fue: Grupo 1: 41\%; Grupo 2: 46\%; Grupo 3: 48\%, y a seis meses fue Grupo 1: 91\%; Grupo 2: 90\%; Grupo 3: 93\%. Medida por el número de pañales/día $(<1$ ó $>1)$. Conclusiones: Después de 100 casos, se mejoran los resultados quirúrgicos tanto en tiempo operatorio como de márgenes quirúrgicos, la tasa de complicaciones y los resultados con la continencia. Basándonos en estos resultados podemos establecer la curva de aprendizaje en 100 procedimientos.

Palabras clave: Cáncer de próstata, prostatectomía radical laparoscópica.

\section{Introducción}

La prostatectomía radical es una de las principales herramientas terapéuticas para el cáncer prostático localizado, y es una de las cirugías urológicas más frecuentes ${ }^{1}$. La técnica quirúrgica más frecuentemente realizada en todas partes del mundo es la prostatectomía radical retropúbica, técnica popularizada en la década de los 80' gracias los trabajos clásicos de Walsh sobre prostatectomía radical y preservación de las bandeletas neurovasculares responsables de la erección ${ }^{2}$. La prostatectomía radical retropúbica es una técnica que puede tener complicaciones intra y postoperatorias de magnitud variable, y no debe ser vista como una cirugía simple. En 1992 Schuessler publicó el primer caso de una prostatectomía radical realizada por vía laparoscópica. Sin embargo, la serie inicial demostró resultados más bien desalentadores ${ }^{3,4}$. En el año 2000, los trabajos de Guillonneau y Vallancien demuestran por primera vez la factibilidad técnica de la prostatectomía radical laparoscópica, realizada por vía transperitoneal, con resultados aceptables y que han sido reproducidos en otros centros alrededor del mundo ${ }^{5-8}$. Con el propósito de imitar la vía extraperitoneal de la prostatectomía radical retropúbica, es que Bollens por primera vez describe esta técnica por una vía exclusiva preperitoneal, con buenos resultados?.

Luego de haber realizado una experiencia inicial en prostatectomía radical laparoscópica por vía transperitoneal, decidimos realizar una experiencia con la técnica extraperitoneal, cuyos resultados reportamos en nuestros primeros 310 casos operados en forma consecutiva.

El objetivo principal de éste trabajo es evaluar la incidencia que tiene la curva de aprendizaje en esta técnica quirúrgica compleja, principalmente en términos de complicaciones, índice de transfusiones y márgenes quirúrgicos oncológicos.

\section{Material y Método}

Entre los años 2001 y 2005, 310 pacientes fueron operados en forma consecutiva. Se consideró candidatos a todos los pacientes portadores de un cáncer prostático clínicamente localizado de indicación 
quirúrgica. Se realizó la técnica de Prostatectomía Radical Laparoscópica Extraperitoneal. Se registraron los datos demográficos y quirúrgicos de todos los pacientes en una base de datos, así como las complicaciones y los resultados de anatomía patológica. Se incluye un análisis de continencia urinaria postoperatoria, evaluando continencia inmediata y a los 3, 6 y 12 meses. Se consideraron continentes los pacientes que requerían 0 paños tanto de día como de noche. En este estudio no se analizan los resultados de función eréctil postoperatoria por no contar con un tiempo de seguimiento suficiente en todos los pacientes. Para efectos del análisis y para establecer la evolución de los resultados quirúrgicos en el tiempo, se dividió la serie en los 100 primeros procedimientos (grupo 1), los 100 siguientes (grupo 2) y los 110 últimos (grupo 3). El análisis estadístico de los resultados se hizo con el test de $\chi^{2}$, comparando los grupos en pares y se consideró estadísticamente significativo un valor $\mathrm{p}<0,05$.

\section{Técnica quirúrgica}

El paciente se coloca en posición de Lloyd-Davis, no siendo necesario colocar sonda nasogástrica ni apoyo en los hombros, ya que no se abrirá el peritoneo y no se utilizará posición de Trendelemburg forzada.

Se realiza una incisión infraumbilical de $2 \mathrm{~cm}$ y se diseca el espacio preperitoneal, por debajo de los rectos, en forma digital. Luego se introduce un balón de disección, con el cual se logra la disección del espacio prevesical, rechazando el peritoneo en forma atraumática. Es fundamental que previo a la disección con balón se haya colocado una sonda vesical para vaciar la vejiga.

En la incisión se coloca un trócar de Hasson de $12 \mathrm{~mm}$, y posteriormente bajo visión los trócares de trabajo en la siguiente posición: 2 trocares de $5 \mathrm{~mm}$ pararectales entre el pubis y el ombligo, un trócar de $5 \mathrm{~mm}$ paraumbilical derecho y un trócar de 12 $\mathrm{mm}$ paraumbilical izquierdo. El cirujano trabaja por los puertos izquierdos, y sólo utiliza los 2 puertos de $5 \mathrm{~mm}$ parectales cuando se necesita triangular para las suturas.

La cirugía comienza con la apertura de la fascia endopélvica bilateral y control del complejo de la vena dorsal con un punto en 8 , de Vycril 0 con aguja CT-1. Luego, se secciona el cuello vesical con el bisturí armónico (Ethicon Endosurgery, Cincinnati, USA), controlando pequeños vasos sangrantes con coagulación bipolar. Los pedículos son controlados con el bisturí armónico, y en caso de preservación de bandeletas la disección procede liberando la fascia periprostática. Se seccionan los conductos deferentes y se liberan las vesículas seminales, y con tijera fría se secciona la hoja posterior de la fascia de Denonvilliers, siguiendo la disección lo más distal posible para liberar el recto.

Luego se diseca el ápex con tijera fría, preservando la mayor longitud de uretra y seccionándola completa. Se termina la prostatectomía seccionado con tijera fría el músculo recto-uretral por el borde postero-lateral. Se coloca la próstata en una bolsa de polietileno, alejándola del campo quirúrgico. El siguiente paso es la anastomosis vesico-uretral, para la cual utilizamos puntos separados de Vycril 2-0, con aguja SH o UR-6. Terminada la anastomosis se coloca una sonda vesical de $22 \mathrm{~F}$ y se extrae la próstata por una pequeña ampliación de la incisión umbilical. Se coloca un drenaje aspirativo a la pelvis y se cierran las incisiones. En los casos en que está indicada linfadenectomía ilio-obturatriz, ésta se realiza posterior a la prostatectomía.

\section{Resultados}

En un período de 5 años se realizaron 310 prostatectomías radicales laparoscópicas extraperitoneales. Las características de los pacientes se resumen en la Tabla 1. La edad promedio de la serie global es 62,4 años (rango de 40 a 78 años), con un score de Gleason (grado histológico) promedio de 6 (rango de 4 a 9), porcentaje de estadío T1c (no palpable) de $69 \%$ con un antígeno prostático específico en sangre (APE) promedio de 13,3. Todas estas características se distribuyeron homogéneamente en los tres grupos analizados.

Respecto de los datos quirúrgicos, el tiempo operatorio promedio fue $184,7,138,9$ y 134,3 minutos para los grupos 1, 2 y 3 respectivamente. El sangrado promedio para los tres grupos fue 451,5, 624,4 y $268,7 \mathrm{ml}$ respectivamente. La tasa de transfusión fue de 20, 22 y 2,7\%. El peso promedio de la glándula resecada fue 46,7, 54,9 y 51,9 gramos. Desde el punto de vista oncológico, el score de Gleason promedio en la pieza quirúrgica fue similar en los tres grupos. El estadio patológico más frecuente en los 3 grupos fue pT2, lo que representó el 41, 40 y $36,3 \%$ en cada grupo. La distribución del resto de los estadíos también fue homogénea, como se aprecia en la Tabla 2. Hubo márgenes quirúrgicos positivos en el 24,7 y $13,6 \%$ de los pacientes en los 3 grupos respectivamente.

Dentro de las complicaciones inmediatas, la más frecuente fue la lesión de la pared rectal anterior, que fue diagnosticada en 9 pacientes del grupo 1 . En 6 casos fue diagnosticada en el intraoperatorio y reparada inmediatamente. En los 3 casos restantes la lesión fue inadvertida y se manifestó como fístula recto-uretral en el postoperatorio tardío. En 
2 casos, de los diagnosticados en el intraoperatorio, la reparación primaria falló y se presentó una fístula tardía. En el grupo 2 se presentaron 2 casos de lesión rectal diagnosticadas y tratadas en el intraoperatorio, y dos casos de lesión inadvertida que se manifestó como fístula rectal en el postope- ratorio. En el grupo 3 no hubo lesiones de la pared rectal. Otra complicación fue la fístula urinaria, que se presentó en 4 pacientes del grupo 1 , en 1 paciente del grupo 2 y en ningún paciente del grupo 3 . Ocho pacientes del grupo 1 y 5 del grupo 2 desarrollaron estenosis de la anastómosis uretro-vesical.

Tabla 1. Características preoperatorios

\begin{tabular}{|c|c|c|c|c|c|c|}
\hline & \multicolumn{2}{|c|}{ Grupo 1} & \multicolumn{2}{|c|}{ Grupo 2} & \multicolumn{2}{|c|}{ Grupo 3} \\
\hline Número & 100 & & 100 & & 110 & \\
\hline Edad promedio (rango) & 63,1 & $(40-76)$ & 62,8 & $(49-78)$ & 61,4 & $(47-78)$ \\
\hline Gleason score promedio (rango) & 6 & $(4-9)$ & 6 & $(4-9)$ & 6 & $(4-9)$ \\
\hline APE promedio (rango) & 11,5 & $(1,8-55,6)$ & 12,1 & $(3,3-52)$ & 16,3 & $(4-25)$ \\
\hline \multicolumn{7}{|l|}{ Estadio clínico } \\
\hline $\mathrm{T} 1 \mathrm{a}$ & 0 & & 0 & & 1 & \\
\hline $\mathrm{T} 1 \mathrm{c}$ & 64 & & 74 & & 76 & \\
\hline $\mathrm{T} 2 \mathrm{a}$ & 27 & & 19 & & 17 & \\
\hline $\mathrm{T} 2 \mathrm{~b}$ & 4 & & 7 & & 6 & \\
\hline $\mathrm{T} 3 \mathrm{a}$ & 2 & & 0 & & 0 & \\
\hline $\mathrm{T} 3 \mathrm{~b}$ & 1 & & 0 & & 0 & \\
\hline Antecedente cirugía abdominal previa & 30 & & 19 & & 8 & \\
\hline RTU previa & 6 & & 5 & & 5 & \\
\hline Hormonoterapia previa & 5 & & 2 & & 7 & \\
\hline
\end{tabular}

Tabla 2. Características operatorias

\begin{tabular}{|c|c|c|c|c|c|c|}
\hline \multirow[b]{2}{*}{ Próstata peso promedio (g) (rango) } & \multicolumn{2}{|c|}{ Grupo 1} & \multicolumn{2}{|c|}{ Grupo 2} & \multicolumn{2}{|c|}{ Grupo 3} \\
\hline & 46,7 & $(14-120)$ & 54,9 & $(22-176)$ & 51,9 & $(15-88)$ \\
\hline Gleason score (rango) & 7 & $(5-9)$ & 7 & $(5-9)$ & 7 & $(6-9)$ \\
\hline \multicolumn{7}{|l|}{ Estadio Patológico } \\
\hline $\mathrm{T} 2 \mathrm{a}$ & 13 & & 13 & & 8 & \\
\hline $\mathrm{T} 2 \mathrm{~b}$ & 41 & & 40 & & 40 & \\
\hline $\mathrm{T} 3 \mathrm{a}$ & 15 & & 14 & & 7 & \\
\hline $\mathrm{T} 3 \mathrm{~b}$ & 12 & & 2 & & 1 & \\
\hline $\mathrm{T} 3 \mathrm{c}$ & 11 & & 10 & & 5 & \\
\hline $\mathrm{T} 4 \mathrm{a}$ & 3 & & & & 1 & \\
\hline Ganglios positivos & $3 / 74$ & $(4 \%)$ & $0 / 18$ & $(0 \%)$ & $2 / 29$ & $(6,8 \%)$ \\
\hline Margen positivo & 24 & $(24 \%)$ & 9 & $(9 \%)$ & 13 & $(11,8 \%)$ \\
\hline \multicolumn{7}{|l|}{ Preservación de bandeletas } \\
\hline Bilateral & 34 & $(34 \%)$ & 56 & $(56 \%)$ & 45 & $(40,9 \%)$ \\
\hline Unilateral & 14 & $(14 \%)$ & 8 & $(8 \%)$ & 8 & $(7,3 \%)$ \\
\hline No preservación & 52 & $(52 \%)$ & 36 & $(36 \%)$ & 57 & $(51,8 \%)$ \\
\hline Tiempo quirúrgico promedio (rango) (min) & 184,7 & $(80-390)$ & 138,9 & $90-180)$ & 134,3 & $(80-310)$ \\
\hline Sangrado promedio (rango) (ml) & 451,5 & $(50-2.000)$ & 624,4 & $(100-2.000)$ & 268,7 & $(20-1.000)$ \\
\hline Transfusión (Promedio Unidades) & $20 \%$ & $(2,2)$ & $22 \%$ & $(2,6)$ & $2,7 \%$ & $(3,5)$ \\
\hline
\end{tabular}


Tabla 3. Complicaciones

\section{Grupo 1 Grupo 2 Grupo 3}

\begin{tabular}{|c|c|c|c|}
\hline \multicolumn{4}{|l|}{ Inmediatas } \\
\hline Lesión de recto & 9 & 2 & 0 \\
\hline Fístula urinaria & 4 & 1 & 0 \\
\hline Tardias & & & \\
\hline Fístula rectal & 5 & 2 & 0 \\
\hline Íleo mecánico & 1 & 0 & 0 \\
\hline $\begin{array}{l}\text { Estenosis cuello } \\
\text { vesical }\end{array}$ & 8 & 5 & 0 \\
\hline Hemoperitoneo & 0 & 1 & 0 \\
\hline $\begin{array}{l}\text { Hematoma } \\
\text { infectado }\end{array}$ & 0 & 1 & 0 \\
\hline Linfocele & 0 & 1 & 0 \\
\hline $\begin{array}{l}\text { Sangrado } \\
\text { postoperatorio }\end{array}$ & 0 & 0 & $\begin{array}{c}1 \\
\text { (Fallece } 45 \text { días) }\end{array}$ \\
\hline
\end{tabular}

En nuestra serie un paciente falleció (grupo 3) en el día 45 del postoperatorio producto de una falla orgánica múltiple que se originó luego de un sangrado postoperatorio, politransfusión y distress respiratorio (Tabla 3 ).

En la Tabla 4 se desglosa el estado de márgenes quirúrgicos de acuerdo al estadio patológico. Se observa que el estadío patológico que con mayor frecuencia dio márgenes positivos es el $\mathrm{pT} 2 \mathrm{~b}$ para los grupos 1 y 2 , mientras en el grupo 3 ocurrió con mayor frecuencia en los tumores pT3a.

En la Tabla 5 se describen los resultados de continencia urinaria. La continencia se logró en el 91, 90 y $93 \%$ de los pacientes de los grupos 1, 2 y 3 respectivamente. Esta fue inmediata (al retiro de la sonda uretral) en el 41, 46 y $48 \%$ respectivamente.

\section{Discusión}

En los últimos 20 años la morbilidad asociada a la prostatectomía radical retropúbica se ha reducido notablemente, debido en gran medida a la estandarización de la técnica gracias a precursores como Walsh, logrando una alta preservación de la continencia y cada vez mejores resultados de preservación de la erección ${ }^{2}$. Por otro lado, existen alternativas que se han demostrado eficaces y segu-
Tabla 4. Margen positivo según estadío patológico

\begin{tabular}{lccc}
\hline & Grupo1 & Grupo 2 & Grupo 3 \\
\hline Estadio patológico & \multicolumn{2}{c}{$N^{o}$ pacientes $/ N^{o}$ margen $+(\%)$} \\
PT2a & 1 & 0 & 1 \\
PT2b & 11 & 5 & 2 \\
PT3a & 2 & 2 & 6 \\
PT3b & 4 & 0 & 1 \\
PT3c & 3 & 2 & 2 \\
PT4a & 3 & 0 & 1 \\
Total & 24 & 9 & 13 \\
& $(24 \%)$ & $(9 \%)$ & $(11,8 \%)$ \\
\hline
\end{tabular}

Tabla 5. Tiempo a continencia completa (0 paños)

\begin{tabular}{lccc}
\hline & $\begin{array}{c}\text { Grupo1 } \\
\mathbf{\%}\end{array}$ & $\begin{array}{c}\text { Grupo 2 } \\
\mathbf{\%}\end{array}$ & $\begin{array}{c}\text { Grupo 3 } \\
\mathbf{\%}\end{array}$ \\
\hline Inmediata & 41 & 46 & 48 \\
Total & 91 & 90 & 93 \\
\hline
\end{tabular}

ras para el tratamiento del cáncer de próstata localizado, como la radioterapia, en constante evolución ${ }^{10}$. En este escenario la prostatectomía radical laparoscópica se introdujo hace varios años como una alternativa mínimamente invasiva a una cirugía probadamente efectiva y segura como la prostatectomía radical retropúbica ${ }^{11}$, razón por la cual ha sido obligada a exhibir altísimos estándares de calidad y seguridad desde el principio. La experiencia inicial de Schuessler no fue alentadora, por lo que la idea introducida en 1992, no logró captar adeptos y fue secundada por muy pocos grupos urológicos en el mundo ${ }^{2,3}$.

Inspirados por la factibilidad técnica de este abordaje demostrada por Guillonneau y Vallancien nuestro grupo comenzó a desarrollar una experiencia similar ${ }^{4,5}$. Luego de una primera experiencia con la técnica transperitoneal, parecía obvio intentar una técnica más parecida a la cirugía abierta practicada por la mayoría de los urólogos, extraperitoneal, y que teóricamente se alejara de las complicaciones propias de las celiotomías.

La experiencia que reportamos ha sido realizada en una población no seleccionada de hombres portadores de cáncer de próstata localizado y candidatos a cualquier alternativa de abordaje quirúrgico, de manera que la evolución de los resultados en el tiempo es manifestación de la evolución de la téc- 
nica. En efecto, no hay diferencias significativas en las características preoperatorias de los pacientes al agruparlos en 3 etapas: Primeros cien, 100 siguientes y últimos 110 .

Hay una disminución evidente y estadísticamente significativa en el tiempo quirúrgico entre el grupo 1 y los grupos 2 y 3 , donde se mantiene estable. El sangrado, aunque presenta una tendencia al alza en el grupo 2, baja significativamente en el último grupo de pacientes $(451,624$ y $268 \mathrm{ml}$ para grupos 1,2 y 3 ), lo que se manifiesta indirectamente en una muy baja tasa de transfusión (2,7\% vs 20\% y $22 \%$ para los grupos 1 y 2 respectivamente). Desde el punto de vista oncológico, hay una evolución hacia disminuir la incidencia de márgenes quirúrgicos positivos. Es interesante notar que esto ocurre teniendo en cuenta que en el primer grupo hubo una tendencia a hacer menos preservación de haces neurovasculares, lo que permite una disección más alejada de la cápsula.

En cuanto a las complicaciones, la más temida especialmente cuando la técnica era transperitoneal, es la lesión del recto. En las series publicadas inicialmente éstas eran manejadas con colostomía independiente de lo satisfactoria que pudiera ser la reparación ${ }^{12}$. Nosotros no hacemos de rutina preparación del colon en el período preoperatorio, y seguimos haciendo reparación primaria sin colostomía cuando advertimos lesiones del recto en el intraoperatorio, lo que es actualmente una tendencia mundial, incluso cuando hemos migrado nuevamente a una técnica transperitoneal. Este manejo ha resultado seguro, tal como lo hemos publicado recientemente ${ }^{13}$. En este estudio se revisa el manejo de 9 pacientes con lesión rectal que corresponden a los primeros 110 pacientes de la serie. De los 6 paciente diagnosticados y reparados intra operatoriamente, 3 tuvieron mejoría sin necesidad de colostomía y los otros 3 pacientes fueron manejados conservadoramente con cateterización uretral durante 30 días. En todos los pacientes con diagnóstico post operatorio (3 pacientes), la reparación se llevó a cabo con un acceso transanal, transesfinteriano sagital, el cual ha permitido un manejo satisfactorio y $\sin$ recurrencias con una media de seguimiento de 24 meses. Además no se reportan pacientes con incontinenecia fecal o estenosis anal, sin afectar la continencia urinaria post prostatectomía ${ }^{14}$.

En nuestra serie reportamos excelentes tasas de continencia, lo que atribuimos a una prolija disección del ápex y de la uretra, gracias a la magnificación del laparoscopio. En el primer grupo hay una alta tasa de estenosis de la anastómosis uretrovesical al compararla con el resto de la serie, aunque no mayor a las publicadas para cirugía abier- ta $^{15}$. Nos parece que esto es una manifestación de la complejidad de la sutura intracorpórea, que es una de las habilidades que tiene una curva de aprendizaje más prolongada, como en toda la laparoscopía urológica reconstructiva.

En nuestra serie sólo tenemos que lamentar el fallecimiento de un paciente, lo que representa una mortalidad totalmente aceptable $(0,32 \%)$, especialmente en una serie de pacientes no seleccionados.

Los buenos resultados obtenidos con la prostatectomía radical laparoscópica extraperitoneal no sólo se deben a las características propias de este abordaje, si no también a que prácticamente todos los grupos que la han desarrollado, incluyéndonos, han hecho experiencias previas en abordaje laparoscópico transperitoneal, por lo que las curvas de aprendizaje no han partido de cero ${ }^{16}$.

En la actualidad hemos vuelto al abordaje transperitoneal, lo que nos ofrece un campo operatorio más amplio y un acceso más expedito, necesarios para facilitar el entrenamiento de nuevos urólogos laparoscopistas. Existen series que comparan los beneficios de los abordajes laparoscópicos extra y transperitoneales, que no demuestran una clara superioridad de un método sobre el otro en términos de resultados y éstos parecen depender de la experiencia de cada grupo ${ }^{17}$.

Dada la historia natural del cáncer de próstata, es necesario esperar varios años antes de establecer en forma definitiva cuales son los resultados en cuanto a control del cáncer en nuestra serie. Sin embargo, tomando en cuenta los resultados quirúrgicos obtenidos, podemos anticipar que éstos no debieran ser diferentes a los resultados oncológicos de la prostatectomía radical retropúbica.

\section{Referencias}

1. Cooperberg M, Broering J, Litwin M, Lubeck D, Metha $\mathrm{S}$, Henning $\mathrm{J}$, et al. The contemporary management of prostate cancer in the United States: Lessons from the cancer of the prostate strategic urologic research endeavour (CAPSURE), a national disease registry. J Urol 2004; 171: 1393-1401.

2. Walsh P. Anatomic radical prostatectomy: evolution of the surgical technique. J Urol 1998; 160(6 Pt 2): 2418-2424.

3. Schuessler W, Kavoussi L, Clayman R. Laparoscopic radical prostatectomy: initial case report. J Urol 1992 suppl; 147: 246A.

4. Schuessler W, Schulam P, Clayman R. Laparoscopic radical prostatectomy: initial short-term experience. Urology 1997; 50: 854-847.

5. Guillonneau B, Cathelineau X, Barret E, Rozet F, Vallancien G. Laparoscopic radical prostatectomy: technical and early oncological assessment of 40 
operations. Eur Urol 1999; 36: 14-20.

6. Guillonneau B, Vallancien G. Laparoscopic radical prostatectomy: the Montsouris experience. J Urol 2000; 163: 418- 422.

7. Jacob F, Salomon L, Hoznek A. Laparoscopic radical prostatectomy: preliminary results. Eur Urol 2000; 37: 615-618.

8. Abbou C, Salomon L, Hoznek A. Laparoscopic radical prostatectomy: preliminary results. Urology 2000; 55: 630-634.

9. Bollens R, Vanden Bossche M, Roumeguere T, Damoun A, Ekane S, Hoffmann P, et al. Extraperitoneal laparoscopic radical prostatectomy. Results after 50 cases. Eur Urol 2001; 4: 65-69.

10. D'Amico AV. Radiation and hormonal therapy for locally advanced and clinically localized prostate cancer. Urology 2002; 60: 37-42.

11. Tooher R, Swindle P, Woo H, Miller J, Maddern G. Laparoscopic Radical Prostatectomy for Localized Prostate Cancer: A Systematic Review of Comparative Studies. J Urol 2006 175: 2011-2017.

12. Stolzenburg JU, Rabenalt R, Do M, Lee B, Truss MC, McNeill A, et al. Complications of endoscopic extraperitoneal radical prostatectomy (EERPE): prevention and management. World J Urol 2006; 24: 668-671.

13. Castillo O, Bodden E, Vitagliano G. Management of Rectal Injury during Laparoscopic Radical Prostatectomy. International Braz J Urol 2006; 32: 428433.

14. Castillo OA, Bodden EM, Vitagliano GJ, Gómez R. Anterior transanal, transsphinteric sagittal approach for fistula repair secondary to laparoscopic radical prostatectomy: A simple and effective technique. Urology 2006; 68: 198-201.

15. Geary ES, Dendinger TE, Freiha FS, Stamey TA. Incontinence and vesical neck strictures following radical retropubic prostatectomy. Urology 1995; 45: 1000-1006.

16. Cathelineau X, Cahill D, Widmer H, Rozet F, Baumert H, Vallancien G. Transperitoneal or Extraperitoneal Approach for Laparoscopic Radical Prostatectomy: A False Debate Over a Real Challenge. J Urol 2004; 171: 714-716.

17. Van Velthoven RF. Laparoscopic radical prostatectomy: transperitoneal versus retroperitoneal approach: is there an advantage for the patient? Curr Opin Urol 2005; 15: 83-88. 